\title{
ON QUANTUM COHOMOLOGY RINGS OF FANO MANIFOLDS AND A FORMULA OF VAFA AND INTRILIGATOR*
}

\author{
BERND SIEBERT ${ }^{\dagger}$ AND GANG TIAN TI $^{\ddagger}$
}

0. Introduction. Quantum multiplications on the cohomology of symplectic manifolds were first proposed by the physicist Vafa [Va2], based on Witten's topological sigma models [Wi1]. In [RuTi], Ruan and the second named author gave a mathematical construction of quantum multiplications on cohomology groups of positive symplectic manifolds (cf. Chapter 1). The definition uses certain symplectic invariants, called Gromov-Witten (GW-) invariants, that were previously defined by Ruan for (semi-) positive symplectic manifolds [Ru]. A large class of such manifolds is provided by Fano manifolds (complex manifolds with ample anti-canonical bundle). Examples are low degree complete intersections and compact complex homogeneous spaces like Grassmann manifolds. If $M$ is a Fano (or positive symplectic) manifold the quantum cohomology $Q H_{[\omega]}^{*}(M)$ is just the cohomology space $H^{*}(M, \mathbb{C})$ with a (non-homogeneous) associative, graded commutative multiplication, the quantum multiplication. This multiplication depends on the choice of a (complexified) Kähler class $[\omega]$ on $M$. Its homogeneous part (the "weak coupling limit" $\lambda \cdot[\omega], \lambda \rightarrow \infty$ ) is the usual cup product.

In this note we observe that quantum cohomology rings have a nice description in terms of generators and relations: If $H^{*}(M, \mathbb{C})=\mathbb{C}\left[X_{1}, \ldots, X_{N}\right] /\left(f_{1}, \ldots, f_{k}\right)$ is a presentation of the cohomology ring (for simplicity we assume $\operatorname{deg} X_{i}$ even for the moment) then $Q H_{[\omega]}^{*}(M)=\mathbb{C}\left[X_{1}, \ldots, X_{N}\right] /\left(f_{1}^{[\omega]}, \ldots, f_{k}^{[\omega]}\right)$, where $f_{1}^{[\omega]}, \ldots, f_{k}^{[\omega]}$ are just the polynomials $f_{1}, \ldots, f_{k}$ evaluated in the quantum ring associated to $[\omega]$ (Theorem 2.2). The $f_{i}^{[\omega]}$ are real-analytic in $[\omega]$ and thus have a natural analytic extension to $H^{1,1}(M)$, and the quantum cohomology rings fit together into a flat analytic family over $H^{1,1}(M)$.

As an application of this observation we shall compute the quantum cohomology of the Grassmannians. The calculations for $\mathrm{G}(k, n)$ reduce to the single quantum product $c_{k} \wedge_{\mathrm{Q}} s_{n-k}$ of the top non-vanishing Chern respectively Segre class of the tautological $k$-bundle. We derive in Chapter 3:

THEOREM 0.1. Let $S$ be the tautological bundle over the Grassmann manifold $\mathrm{G}(k, n)$ of complex $k$-planes in $\mathbb{C}^{n},[\omega]=-\lambda \cdot c_{1}(S)$ a $(1,1)$-class on $\mathrm{G}(k, n)(\lambda \in$ $\mathbb{R}_{>0} \Leftrightarrow[\omega]$ Kähler $)$. Then

$$
Q H_{[\omega]}^{*}(\mathrm{G}(k, n))=\mathbb{C}\left[X_{1}, \ldots, X_{k}\right] /\left(Y_{n-k+1}, \ldots, Y_{n-1}, Y_{n}+(-1)^{k} e^{-\lambda}\right),
$$

where $X_{i}$ corresponds to the $i$-th Chern class $c_{i}(S)$ and the $Y_{j}$ (corresponding to the $j$-th Segre class of $S)$ are given recursively by $\left(X_{i}=0\right.$ for $\left.i>k\right)$

$$
Y_{j}=-Y_{j-1} \cdot X_{1}-\ldots-Y_{1} \cdot X_{j-1}-X_{j}
$$

This is in fact the form previously derived by Vafa using arguments from Quantum Field Theory [Va1], [Va2] (but note the sign of the quantum contribution). These

\footnotetext{
${ }^{*}$ Received September 22, 1997; accepted for publication November 25, 1997.

†Fakultät für Mathematik, Ruhr-Universität Bochum, D-44780 Bochum, Germany (Bernd. Siebert@ruhr-uni-bochum.de).

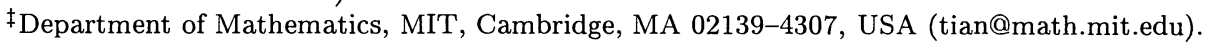


papers and [In] also contain an intriguing formula for all GW-invariants (of the type considered here) of Grassmannians. We will see in Chapter 4 that formulas of this type occur whenever the cohomology ring has a presentation as complete intersection. In particular, we prove as Corollary 4.6

THEOREM 0.2. (Vafa-Intriligator formula) For any Kähler class $[\omega]$ there is a finite set $C \subset \mathbb{C}^{n}$ and non-zero constants $a_{x}, x \in C$, such that for any $F \in$ $\mathbb{C}\left[X_{1}, \ldots, X_{k}\right]$

$$
\langle F\rangle_{g}^{[\omega]}=\sum_{x \in C}\left(a_{x}\right)^{g-1} \cdot F(x)
$$

Here $\langle F\rangle_{g}^{[\omega]}$ is the genus $g G W$-invariant associated to $F$. In fact, there is a polynomial $W^{[\omega]}$ in the $X_{i}$ having $C$ as its set of critical points with $a_{x}$ being (up to sign) the determinants of the Hessians at $x \in C$.

Some remarks on history and related works are in order. One of our starting points was a paper by Bertram, Daskalopoulos and Wentworth giving a mathematical formulation of the Vafa-Intriligator formula in terms of intersection theory on certain compactifications of moduli spaces of maps from a (fixed) Riemann surface to $\mathrm{G}(k, n)$ [BDW]. They verified the formula for genus one and $k=2$. The content of the first three chapters was known to the authors around New Year 93/94 and a preliminary version of the paper was spread in January 1994. A preprint version of the full paper was made available to the public as alg-geom/940310. The presentation theorem for quantum cohomology rings has been observed independently in [AsSa]. Some of the considerations of Chapter 2 appear also in [Pi]. We also realized later that the quantum product $c_{k} \wedge s_{n-k}$ had already been computed in [Wi3], up to a check of genericity conditions. His use of residues for the interpretation of coefficients of quantum products inspired us to the arguments in Chapter 4. The main technical result there (Proposition 4.1) about higher dimensional residues has independently been obtained in [CaDiSt].

Further partial support for the Vafa-Intriligator formula was obtained in [RRW] and [Be1]. The quantum cohomology of (absolute) flag manifolds (containing $\mathrm{G}(k, n)$ as special case) has been calculated in [GiKi] (complete flags) and [AsSa] (general case) assuming the existence of an equivariant version of quantum cohomology with certain functorial properties. A description (and a proof of associativity) of the quantum multiplication for Grassmannians in terms of Schubert cycles ("quantum Pieri" and "quantum Giambelli" formulae) has later been obtained by Bertram [Be2].

By the effort of several people the quantum cohomology of generalized flag manifolds $G / B$ have more recently been computed in complete generality, cf. [Ki] and the references given there.

The first named author thanks the DFG for support and the Courant Institute for hospitality during the academic year 1993/1994 where this work has been done. The second author is partly supported by an NSF grant and an Alfred P. Sloan Fellowship.

1. Definition of quantum multiplications. In this section, we recall the definition of quantum multiplications for positive manifolds as given in [RuTi]. The definition uses the $\mathrm{GW}$-invariants from $[\mathrm{Ru}]$.

A symplectic manifold $(M, \omega)$ of dimension $2 n$ is called positive if for any $f$ : $\mathbb{P}^{1} \rightarrow M$ with $[\omega](R)>0$ it holds $c_{1}(M)(R)>0, R=f_{*}\left[\mathbb{P}^{1}\right] \in H_{2}(M, \mathbb{Z})$. Example: Fano manifolds are positive with respect to a Kähler form representing $c_{1}(M)$. Let $J$ 
be an almost complex structure on $M$. A (parametrized) $J$-holomorphic curve on $M$ is a smooth map $f: \Sigma \rightarrow M$ from a Riemann surface $\Sigma$ satisfying

$$
J \circ D f=D f \circ j,
$$

as morphisms from $T_{\Sigma}$ to $f^{*} T_{M}$, where $j$ is the complex structure of $\Sigma$. This equation is a Cauchy-Riemann equation

$$
\bar{\partial}_{J} f=0, \quad \bar{\partial}=\frac{1}{2}(D+J \circ D \circ j) .
$$

For a technical reason one also needs the inhomogeneous equation $\bar{\partial}_{J} f=\nu$ with $\nu$ a section of $\Lambda^{0,1} \otimes_{\mathbb{C}} T_{M}$ over $\Sigma \times M, \Lambda^{0,1}$ the bundle of $(0,1)$-forms on $\Sigma$. Solutions of this equation are called perturbed or $(J, \nu)$-holomorphic curves. The symplectic structure comes in by the assumption that $J$ be tamed by $\omega$ which by definition means

$$
\omega(X, J X)>0 \quad \forall X \in T_{M} \backslash\{0\} .
$$

Without this assumption moduli spaces of $J$-holomorphic curves can have rather bad non-compactness behaviour. The tameness condition is crucial in both defining GWinvariants and showing that they are invariants of the symplectic structure.

GW-invariants depend on the choice of $R \in H_{2}(M, \mathbb{Z})$ and integral homology classes $B_{1}, \ldots, B_{s} \in H_{*}(M, \mathbb{Z}$ ) satisfying ( $g$ is the genus of $\Sigma$ ):

$$
\sum_{i=1}^{s}\left(2 n-\operatorname{deg} B_{i}\right)=2 c_{1}(M)(R)+2 n(1-g)=: d(M, R, g) .
$$

Every integral homology class can be represented by so-called pseudo-manifolds (compact subspaces with singularities in real codimension at least 2, cf. [RuTi, Def.5.1]). For simplicity, we shall also use $B_{i}$ to denote the pseudo-manifolds representing these homology classes. Let $t_{1}, \ldots, t_{s} \in \Sigma$ be fixed points. It is shown in [RuTi] that if $(J, \nu)$ is generic the moduli space $\mathcal{M}_{R}(\Sigma, J, \nu)$ of $(J, \nu)$-holomorphic maps of homology class $R$ is smooth of dimension $d(M, R, g)$ (dim) and the boundary of the image of the $s$-fold evaluation map

$$
\text { ev }: \mathcal{M}_{R}(\Sigma, J, \nu) \rightarrow M^{s}, \quad f \longmapsto\left(f\left(t_{1}\right), \ldots, f\left(t_{s}\right)\right)
$$

has lower dimension. Choosing $B_{i}$ transversal to the evaluation map and to the compactifying boundary there are only finitely many $f \in \mathcal{M}_{R}(\Sigma, J, \nu)$ with $f\left(t_{i}\right) \in B_{i}$. A GW-invariant is defined by

$$
\tilde{\Phi}_{(R, \omega)}^{g}\left(B_{1}, \ldots, B_{s}\right):=\sharp\left\{f \in \mathcal{M}_{R}(\Sigma, J, \nu) \mid f\left(t_{i}\right) \in B_{i}\right\},
$$

the algebraic sum of such $f$ counted with appropriate signs according to the canonically given orientation. One can prove that $\tilde{\Phi}_{(R, \omega)}^{g}\left(B_{1}, \ldots, B_{s}\right)$ is independent of the choices of $J, j, \nu$, and pseudo-manifolds representing $B_{1}, \ldots, B_{s}$. As a matter of notation we set $\tilde{\Phi}_{(R, \omega)}^{g}\left(B_{1}, \ldots, B_{s}\right)=0$ unless the dimensions match (dim).

To describe the compactifying set one generalizes the notion of $(J, \nu)$-holomorphic maps to singular domains. Namely, one replaces $\Sigma$ by a connected complex curve $C$ with at most ordinary double points consisting of $\Sigma$ and a number of spheres. One requires the spheres to be attached in such a way that the arithmetic genus of $C$ still equals $g$. So $C$ is obtained from $\Sigma$ by attaching trees of spheres at points $P_{a} \in \Sigma$. A 
$(J, \nu)$-holomorphic map from $C$ to $M$ is then a continuous map $f: C \rightarrow M$ that is $(J, \nu)$-holomorphic on $\Sigma$ and $J$-holomorphic on each of the spheres. The compactifying set is obtained by the image of the obvious extension of the evaluation map, where the $t_{i}$ may now be replaced by any point on a tree of spheres whenever the tree is attached at the original $t_{i}$.

We now make a simple but important remark: Let $J$ be an almost complex structure such that any $J$-holomorphic curve with $f_{*}[C]=R$ is regular, i.e. $C=\Sigma$ is irreducible and the linearization of the Cauchy-Riemann operator at $f$ is surjectice. It follows from the construction of [Ru], [RuTi] that one can use exact $J$-holomorphic curves to compute the invariants $\tilde{\Phi}_{(R,\{\omega\})}^{g}$, cf. [RuTi, Rem.2.8]. In case $J$ is integrable and $f$ is an immersion, regularity at $f$ is equivalent to the vanishing of $H^{1}\left(\Sigma, f^{*} T_{M} / T_{\Sigma}\right)$ [Gr, 2.1.B]. We adopt the convention that a rational curve in a complex manifold is a holomorphic curve (not necessarily smooth or irreducible) of arithmetic genus 1 . The statement used for computation of GW-invariants in this paper is:

Lemma 1.1. Let $(M, \omega)$ be a Kähler manifold and $R \in H_{2}(M, \mathbb{Z})$ such that any rational curve $C \subset M$ homologous to $R$ is non-singular and has $H^{1}\left(C, N_{C \mid X}\right)=0$. Let $B_{1}, B_{2}, B_{3} \subset M$ be complex submanifolds transversal to the (3-fold) evaluation map. Then

$$
\tilde{\Phi}_{(R,\{\omega\})}^{0}\left(B_{1}, B_{2}, B_{3}\right)=\sum_{C}\left(B_{1} \cap C\right) \cdot\left(B_{2} \cap C\right) \cdot\left(B_{3} \cap C\right),
$$

where the sum is over all rational curves $C$ homologous to $R$.

Proof. Let $I$ be the integrable complex structure of $M$. Then $\mathcal{M}_{R}\left(\mathbb{P}^{1}, I, 0\right)$ is nothing but the complex space $\operatorname{Hom}_{R}\left(\mathbb{P}^{1}, M\right)$ of holomorphic maps $\mathbb{P}^{1} \rightarrow M$ which is thus smooth. And by the Gromov compactness theorem and the assumption on smoothness of all rational curves representing $R, \mathcal{M}_{R}\left(\mathbb{P}^{1}, I, 0\right)$ is compact. Furthermore $\left(B_{1} \cap C\right) \ldots\left(B_{3} \cap C\right)$ is precisely the number of ways to parametrize $C$ by $f: \mathbb{P}^{1} \rightarrow C$ with three distinguished points $t_{i} \in \mathbb{P}^{1}$ mapping to $B_{i}, i=1,2,3$. Finally there are no signs occurring in the formula since all spaces involved are canonically oriented by their complex structures.

To define a quantum multiplication on $H^{*}(M, \mathbb{Z})$, we introduce the real-valued invariant

$$
\tilde{\Phi}_{[\omega]}^{g}\left(B_{1}, \ldots, B_{s}\right)=\sum_{R \in H_{2}(M, \mathbb{Z})} \tilde{\Phi}_{(R,\{\omega\})}^{g}\left(B_{1}, \ldots, B_{s}\right) e^{-[\omega](R)} .
$$

In general there might be infinitely many terms contributing to the sum (e.g. in the important and interesting case of Calabi-Yau manifolds) and one faces a nontrivial convergence problem. In the positive case, however, this sum is actually finite due to the dimension condition (dim). Let us assume $M$ positive for the following (but see Remark 2.3). Note also that letting $\omega$ vary one gets back all the invariants $\tilde{\Phi}_{(R,\{\omega\})}^{g}\left(B_{1}, \ldots, B_{s}\right)$ by solving some system of linear equations. The quantum multiplication $\wedge_{\mathrm{Q}}$ on $H^{*}(M, \mathbb{R})$ is then characterized by the equation

$$
\left(\alpha \wedge_{\mathrm{Q}} \beta\right)[A]=\tilde{\Phi}_{[\omega]}^{0}\left(\alpha^{\vee}, \beta^{\vee}, A\right),
$$

where $\alpha, \beta \in H^{*}(M, \mathbb{Z})$ and $\vee$ means Poincaré-dual. In terms of a basis $\left\{A_{i}\right\}$ for $H_{*}(M, \mathbb{Z})$ modulo torsion and with $\left\{\alpha_{i}\right\}$ the Poincaré-dual basis of $H^{*}(M, \mathbb{Z})$ we may 
state this more explicitly as

$$
\alpha_{i} \wedge_{Q} \alpha_{j}=\sum_{k, l} \eta^{l k} \tilde{\Phi}_{[\omega]}^{0}\left(A_{i}, A_{j}, A_{k}\right) \alpha_{l}
$$

with $\left(\eta^{l k}\right)_{k l}$ inverse to the intersection matrix $\left(\eta_{i j}\right)=\left(A_{i} \cdot A_{j}\right)\left(\sum_{j} \eta_{i j} \eta^{j k}=\delta_{i k}\right)$. The associativity of the quantum multiplication is highly non-trivial and shown by a careful analysis of the degeneration of rational curves in [RuTi] (following an idea of Witten).

An obvious, whence decisive feature of this definition, that we are going to exploit, is that its homogeneous part of highest degree reduces to the cup product

$$
\alpha_{i} \wedge \alpha_{j}=\sum_{k, l} \eta^{l k} \cdot\left(A_{i} \cdot A_{j} \cdot A_{k}\right) \alpha_{l} .
$$

2. A presentation for quantum cohomology. For a commutative ring $A$ and degrees $d_{i}$ attached to variables $X_{i}$ we denote by $A\left\langle X_{1}, \ldots, X_{n}\right\rangle$ the free graded commutative $A$-algebra with generators $X_{i}$ of degree $d_{i}$, i.e. with $X_{i} X_{j}=(-1)^{d_{i} \cdot d_{j}} X_{j} X_{i}$. If $m$ of the $X_{i}$ have odd degree this is isomorphic to $\Lambda^{\bullet} A^{m} \otimes S^{\bullet} A^{n-m}$. We will call elements of this algebra ordered polynomials (since in addition to the coefficients one has to select an order among the factors in a monomial to determine its sign).

Let $(M, \omega)$ be a positive symplectic manifold and

$$
H^{*}(M, \mathbb{Q})=\mathbb{Q}\left\langle X_{1}, \ldots, X_{n}\right\rangle /\left(f_{1}, \ldots, f_{k}\right)
$$

be a homogeneous presentation of the cohomology ring, $f_{i}=\sum_{|J|=\operatorname{deg} f_{i}} a_{i J} X^{J}$. We use multiindex notation $J=\left(j_{1}, \ldots, j_{n}\right), X^{J}=X_{1}^{j_{1}} \wedge \ldots \wedge X_{n}^{j_{n}},|J|=\sum_{i=1}^{n} j_{i} d_{i}$ etc. Denote by $\wedge_{\mathrm{Q}}$ the product in the quantum cohomology $Q H_{[\omega]}^{*}(M)$. To distinguish clearly between calculations in $H^{*}(M)$ and in $Q H_{[\omega]}^{*}(M)$, we use a hat to mark elements of the quantum cohomology. So ${ }^{-}$might be thought of as a $\mathbb{C}$-linear map $\mathbb{C}\left\langle X_{1}, \ldots, X_{n}\right\rangle \rightarrow Q H_{[\omega]}^{*}(M)$. An ordered polynomial $f$ may be evaluated on the generators of the quantum cohomology. We write $f\left(\hat{X}_{1}, \ldots, \hat{X}_{n}\right)$ as in the commutative case. For instance, $\hat{X}^{J}:=\hat{X}_{1} \wedge_{\mathrm{Q}} \ldots \wedge_{\mathrm{Q}} \hat{X}_{1} \wedge_{\mathrm{Q}} \ldots \wedge_{\mathrm{Q}} \hat{X}_{n} \wedge_{\mathrm{Q}} \ldots \wedge_{\mathrm{Q}} \hat{X}_{n}$ with $\hat{X}_{\nu}$ occurring $j_{\nu}$-often (note the difference to $\left.\left(X^{J}\right)^{\wedge} !\right)$.

LEMMA 2.1. $\hat{X}_{1}, \ldots, \hat{X}_{n}$ generate $Q H_{[\omega]}^{*}(M)$.

Proof. By induction on the degree. So assume $\hat{X}_{1}, \ldots, \hat{X}_{n}$ generate $Q H_{[\omega]}^{*}(M)$ up to degree $d-1$. We want to show that the monomials $\left(X^{J}\right)^{\wedge},|J|=d$, can be written as linear combinations of (quantum) products of the $\hat{X}_{\nu}$. But by definition of the multiplication in $Q H_{[\omega]}^{*}(M)$

$$
\hat{X}^{J}=\left(X^{J}\right)^{\wedge}+\sum_{|I|<d} a_{I}\left(X^{I}\right)^{\wedge},
$$

and by induction hypothesis $\left(X^{I}\right)^{\wedge}=\sum_{K \leq I} b_{I K} \hat{X}^{K}$, so

$$
\left(X^{J}\right)^{\wedge}=\hat{X}^{J}-\sum_{\substack{K \leq I \\|I|<d}} a_{I} b_{I K} \hat{X}^{K} .
$$

Next we evaluate $f_{i}\left(\hat{X}_{1}, \ldots, \hat{X}_{n}\right)$, i.e. in the quantum cohomology ring. Since $f_{i}$ is a relation in the cohomology ring, the term of top-degree $\operatorname{deg} f_{i}$ vanishes. By 
the lemma we find an ordered polynomial $g_{i}^{[\omega]}$ (depending on $[\omega]$ ) in $n$ variables (say $\left.T_{1}, \ldots, T_{n}, \operatorname{deg} T_{i}=\operatorname{deg} X_{i}\right)$ of lower degree with

$$
f_{i}\left(\hat{X}_{1}, \ldots, \hat{X}_{n}\right)=g_{i}^{[\omega]}\left(\hat{X}_{1}, \ldots, \hat{X}_{n}\right) \quad \text { in } Q H_{[\omega]}^{*}(M)
$$

Thus $f_{i}^{[\omega]}\left(T_{1}, \ldots, T_{n}\right):=f_{i}\left(T_{1}, \ldots, T_{n}\right)-g_{i}^{[\omega]}\left(T_{1}, \ldots, T_{n}\right) \in \mathbb{C}\left\langle T_{1}, \ldots, T_{n}\right\rangle$ is a nontrivial relation between $\hat{X}_{1}, \ldots, \hat{X}_{n}$.

Proposition 2.2. $Q H_{[\omega]}^{*}(M)=\mathbb{C}\left(T_{1}, \ldots, T_{n}\right\rangle /\left(f_{1}^{[\omega]}, \ldots, f_{k}^{[\omega]}\right)$.

Proof. Let $\mathcal{J} \subset \mathbb{C}\left\langle T_{1}, \ldots, T_{n}\right\rangle$ be the ideal of relations between the $\hat{X}_{1}, \ldots, \hat{X}_{n}$. Then $\left(f_{1}^{[\omega]}, \ldots, f_{k}^{[\omega]}\right) \subset \mathcal{J}$ and $Q H_{[\omega]}^{*}(M)=\mathbb{C}\left\langle T_{1}, \ldots, T_{n}\right\rangle / \mathcal{J}$ by the lemma. Let $F \in \mathcal{J} \backslash\{0\}$. Expand $F=F_{d}+F^{\prime}$ with $F_{d} \neq 0$ (weighted) homogeneous of degree $d$, $d>0$, and $\operatorname{deg} F^{\prime}<d$. Then

$$
F_{d}\left(\hat{X}_{1}, \ldots, \hat{X}_{n}\right)=-F^{\prime}\left(\hat{X}_{1}, \ldots, \hat{X}_{n}\right)
$$

in quantum cohomology, for $F$ is a relation. But the highest degree (deg $=d$ ) contribution to $F_{d}\left(\hat{X}_{1}, \ldots, \hat{X}_{n}\right)$ is just $\left(F_{d}\left(X_{1}, \ldots, X_{n}\right)\right)^{\wedge}$. Its vanishing implies $F_{d} \in$ $\left(f_{1}, \ldots, f_{k}\right)$, i.e. $F_{d}=\varphi\left(f_{1}, \ldots, f_{k}\right), \varphi$ a polynomial in $k$ variables. Thus

$$
\varphi\left(f_{1}^{[\omega]}, \ldots, f_{k}^{[\omega]}\right)=F_{d}+F^{\prime \prime} \quad \text { in } \mathbb{C}\left\langle T_{1}, \ldots, T_{n}\right\rangle
$$

with $\operatorname{deg} F^{\prime \prime}<d$, and we may write $F=\varphi\left(f_{1}^{[\omega]}, \ldots, f_{k}^{[\omega]}\right)+F^{\prime}-F^{\prime \prime}, \operatorname{deg} F^{\prime}-F^{\prime \prime}<$ $d=\operatorname{deg} F$. Proceeding by induction on the degree we finally see $F \in\left(f_{1}^{[\omega]}, \ldots, f_{k}^{[\omega]}\right)$, i.e. $\mathcal{J}=\left(f_{1}^{[\omega]}, \ldots, f_{k}^{[\omega]}\right)$ as claimed.

REMARK 2.3. 1) The proposition is constructive. The induction process shows that a particular coefficient of the perturbed relations $f^{[\omega]}$ may involve several GWinvariants. So the actual computation of $f^{[\omega]}$ is not always easy, even if one knows a good deal about the GW-invariants of $M$. The converse problem of determining $\mathrm{GW}$-invariants from the presentation can be dealt with by Gröbner basis methods readily accessible to computer algebra programs, cf. Chapter 4.

2) In the positive case $c_{1}(M)>0$ the contributions with $R \neq 0$ give rise to terms of lower degree by the dimension condition, so we were able to fix $[\omega]$ and argue by considerations on the degree. Modulo convergence problems mentioned at the end of the previous chapter, Proposition 2.2 remains valid in the semi-positive case. Arguments involving the degree can be replaced by linear independence of terms $e^{-\lambda \cdot t}$ for various $\lambda$ in the algebra $\mathbb{C}\{t\}$.

Another approach, which works without any positivity condition, is to use formal power series over $H_{2}(M, \mathbb{Z}$ ) (cf. also [Pi]): Let $\mathcal{R C}(M) \subset H$ be the monoid generated by classes of $J$-holomorphic rational curves. Since $J$ is also $\omega^{\prime}$-tame for $\omega^{\prime}$ a symplectic form sufficiently close to $\omega$ and $\omega^{\prime}(\mathcal{R C}(M)) \subset \mathbb{R}_{>0}, \mathcal{R C}(M)$ is a strictly convex cone. Multiplication on the group $N:=\mathbb{Z} \llbracket \mathcal{R C}(M) \rrbracket$ of formal power series over $\mathcal{R C}(M)$ is thus well-defined. Then $H^{*}(M, \mathbb{Z}) \otimes_{\mathbb{Z}} N$ appears as natural domain of definition of quantum products for more general manifolds. Our arguments provide a presentation of the quantum cohomology ring as quotient of $\mathbb{Q}\left\langle T_{1}, \ldots, T_{n}\right\rangle \otimes_{\mathbb{Q}} N$ by ordered polynomials with coefficients in $N$. Note also that in the positive case $Q H_{[\omega]}^{*}(M)$ may be viewed as homeomorphic image of $H^{*}(M, \mathbb{Z}) \otimes_{\mathbb{Z}} \mathbb{Z}[\mathcal{R C}(M)] \subset H^{*}(M, \mathbb{Z}) \otimes_{\mathbb{Z}} N$ by sending $R \in H_{2}(M, \mathbb{Z})$ to $e^{-[\omega](R)}$. 
3. Grassmann manifolds. As is well-known (e.g. [Fu, Ex. 14.6.6]), the cohomology ring (in fact even the Chow ring) of the Grassmann variety $\mathrm{G}(k, n)$ of $k$-planes in $\mathbb{C}^{n}$ has a presentation

$$
H^{*}(\mathrm{G}(k, n), \mathbb{Q})=\mathbb{Q}\left[c_{1}, \ldots, c_{k}\right] /\left(s_{n-k+1}, \ldots, s_{n}\right),
$$

with $c_{i}$ corresponding to the Chern classes of the tautological $k$-bundle $S$ and $s_{j}$ to its Segre classes viewed as polynomials in $c_{1}, \ldots, c_{k}$ via

$$
\begin{gathered}
\left(1+c_{1}+\ldots+c_{k}\right)\left(1+s_{1}+s_{2}+\ldots\right)=1, \\
\text { i.e. } s_{j}=-s_{j-1} c_{1}-\ldots-s_{1} c_{j-1}-c_{j} .
\end{gathered}
$$

Note that $s_{j}$ is also the $j$-th Chern class of the universal quotient bundle $Q$, which has rank $n-k$, so $s_{j}=0$ in $H^{*}(\mathrm{G}(k, n))$ for $j>n-k$. In fact, as a polynomial in $c_{i}, s_{j}$ lies in the relation ideal for $j>n$ by the recursion formula $(*)$. Under the canonical isomorphism $\Phi: \mathrm{G}(k, n) \simeq \mathrm{G}(n-k, n), \Lambda \mapsto\left(\mathbb{C}^{n} / \Lambda\right)^{*}, S$ corresponds to the dual of the universal quotient bundle $Q^{\prime}$ on $\mathrm{G}(n-k, n)$ and $Q$ to the dual of the tautological $(n-k)$-bundle $S^{\prime}$. Thus $c_{i}$ and $s_{j}$ exchange (up to sign) their roles and one might as well write

$$
H^{*}(\mathrm{G}(k, n), \mathbb{Q})=\mathbb{Q}\left[s_{1}, \ldots, s_{n-k}\right] /\left(c_{k+1}, \ldots, c_{n}\right),
$$

this time with $c_{i}$ polynomials in $s_{1}, \ldots, s_{n-k}$ (this presentation is actually better adapted to Schubert calculus, i.e. geometry, see below). These remarks are made to emphasize the symmetry between $c_{i}$ and $s_{j}$. Note also that the generators all have even degree, so we need not worry about questions of sign.

Schubert calculus (e.g. [Fu, $\S 14.7])$ provides a basis of $H^{*}(\mathrm{G}(k, n))$ as $\mathbb{Q}$-vector space (indeed a basis of integral homology/cohomology as $\mathbb{Z}$-module), indexed by tuples $\left(\lambda_{1}, \ldots, \lambda_{k}\right), n-k \geq \lambda_{1} \geq \ldots \geq \lambda_{k} \geq 0$, via

$$
\left\{\lambda_{1}, \ldots, \lambda_{k}\right\}:=\operatorname{det}\left(s_{\lambda_{i}+j-i}\right)_{1 \leq i, j \leq k},
$$

i.e. by evaluating the Schur polynomial (S-function) associated to $\left(\lambda_{1}, \ldots, \lambda_{k}\right)$ on the Segre classes of the tautological $k$-bundle $\left(s_{j}=0\right.$ for $\left.j \notin\{0, \ldots, n-k\}\right) .\left\{\lambda_{1}, \ldots, \lambda_{k}\right\}$ is (weighted) homogeneous of degree $2 \sum \lambda_{i}$. The Chern respectively Segre classes are given by

$$
\begin{aligned}
c_{i} & \left.=(-1)^{i}\{1, \ldots, 1,0, \ldots, 0\} \quad \text { ("1" } i \text {-times }\right), \\
s_{j} & =\{j, 0, \ldots, 0\}
\end{aligned}
$$

(for $c_{i}$ see [Fu, Lemma 14.5.1] or do an easy induction). The connection to classical Schubert calculus is given by Poincaré-duality. In fact, $\left\{\lambda_{1}, \ldots, \lambda_{k}\right\} \cap[\mathrm{G}(k, n)]$ may be represented by the Schubert varieties

$$
\Omega_{\underline{V}}\left(n-k+1-\lambda_{1}, \ldots, n-k+i-\lambda_{i}, \ldots, n-\lambda_{k}\right) .
$$

To define the latter one has to fix a flag $\underline{V}=\left(V_{1}, \ldots, V_{n}\right), V_{1} \subset \ldots \subset V_{n}=\mathbb{C}^{n}$ of linear subspaces of $\mathbb{C}^{n}, \operatorname{dim} V_{i}=i$. Then for $\left(a_{1}, \ldots, a_{k}\right), 0 \leq a_{1}<\ldots<a_{k} \leq n$

$$
\Omega_{\underline{V}}\left(a_{1}, \ldots, a_{k}\right):=\left\{\Lambda \in \mathrm{G}(k, n) \mid \operatorname{dim} \Lambda \cap V_{a_{i}} \geq i, 1 \leq i \leq k\right\} .
$$

The homology class $\left(a_{1}, \ldots, a_{n}\right):=\left[\Omega_{\underline{V}}\left(a_{1}, \ldots, a_{k}\right)\right]$ is independent of the choice of flag. We write $\{\underline{\lambda}\}^{\vee}:=\left(n-k+1-\lambda_{1}, \ldots, n-k+i-\lambda_{i}, \ldots, n-\lambda_{k}\right) \in H_{*}(\mathrm{G}(k, n))$. 
What is important for us is that $[\mathrm{G}(k, n)]$ is Poincaré-dual to $\{0, \ldots, 0\}=1$ (trivial) and that the class of a point [*] is Poincaré-dual to $\{n-k, \ldots, n-k\}=s_{n-k}^{k}=$ $(-1)^{n-k} c_{k}^{n-k}$ (which makes sense as $\operatorname{dim} \mathrm{G}(k, n)=k(n-k)$, but is less trivial: What is the Poincaré-dual of $\left.c_{1}^{k(n-k)} ?\right)$. The intersection of classes of complementary dimension is especially easy: $|\underline{\lambda}|+|\underline{\mu}|=k(n-k)$, then ("duality theorem"):

$$
(\{\underline{\lambda}\} \wedge\{\underline{\mu}\})[G]=\{\underline{\lambda}\} \cap\{\underline{\mu}\}^{\vee}=\{\underline{\lambda}\}^{\vee} \cdot\{\underline{\mu}\}^{\vee}= \begin{cases}1 & , \quad \text { if } \underline{\mu}=\underline{\lambda}^{*} \\ 0, & \text { otherwise. }\end{cases}
$$

with $\underline{\lambda}^{*}:=\left(n-k-\lambda_{k}, \ldots, n-k-\lambda_{1}\right)$. In particular, we get $\eta_{\underline{\lambda} \mu}=\delta_{\underline{\lambda}} \underline{\mu}$ for the intersection matrix. This ends our collection of facts concerning Grassmannians.

We thus see that $H^{1,1}(\mathrm{G}(k, n))$ is spanned by the single class $\{1,0, \ldots, 0\}=$ $s_{1}=-c_{1}$. The ample generator is $s_{1}=c_{1}(Q)$ for $s_{1}=\iota^{*} c_{1}\left(\mathcal{O}_{\mathbb{P}\left(\Lambda^{k} \mathbb{C}^{n}\right)}(1)\right)$ where $\iota: \mathrm{G}(k, n) \hookrightarrow \mathbb{P}\left(\Lambda^{k} \mathbb{C}^{n}\right)$ is the Plücker embedding. Dually, $H_{2}(\mathrm{G}(k, n), \mathbb{Z})$ is spanned by the class of a line $\{n-k, \ldots, n-k, n-k-1\}^{\vee}=(1, \ldots, k-1, k+1)=$ : $[L]$. Since $T_{\mathrm{G}(k, n)} \simeq \operatorname{Hom}(S, Q) \simeq S^{*} \otimes Q$, as one easily verifies by using the standard local coordinates on $\mathrm{G}(k, n), c_{1}(\mathrm{G}(k, n))=\operatorname{rk}(Q) \cdot c_{1}\left(S^{*}\right)+\operatorname{rk}\left(S^{*}\right) \cdot c_{1}(Q)=n \cdot s_{1}$.

So $\mathrm{G}(k, n)$ is a Fano manifold of index $n$ with Picard group infinite cyclic of rank one and it makes sense to talk about its quantum cohomology in the version presented in Chapter 1. The product in $Q H_{[\omega]}^{*}(\mathrm{G}(k, n))$ reads

$$
\widehat{\{\underline{\lambda}\}} \wedge_{\mathrm{Q}} \widehat{\{\underline{\mu}\}}=\sum_{R=d \cdot[L], d \geq 0} \sum_{\underline{\nu}} \tilde{\Phi}_{R}^{0}\left(\{\underline{\lambda}\}^{\vee},\{\underline{\mu}\}^{\vee},\{\underline{\nu}\}^{\vee}\right) \widehat{\left\{\underline{\nu}^{*}\right\}} e^{-[\omega](R)}
$$

Recall from Chapter 1 that $\tilde{\Phi}_{d \cdot[L]}^{0}\left(\{\underline{\lambda}\}^{\vee},\{\underline{\mu}\}^{\vee},\{\underline{\nu}\}^{\vee}\right)=0$ unless $|\underline{\lambda}|+|\underline{\mu}|+|\underline{\nu}|=$ $k(n-k)+c_{1}(\mathrm{G}(k, n))(d \cdot[L])=k(n-k)+d \cdot n$. In calculating $s_{n-k+i}\left(\hat{c}_{1}, \ldots, \hat{c}_{k}\right)$ (i.e. in $Q H_{[\omega]}^{*}(\mathrm{G}(k, n)), \hat{c}_{i}$ the generators corresponding to $c_{i}$ as in Chapter 2) we have $|\underline{\lambda}|+|\underline{\mu}| \leq n$, and "=" only in the case of $s_{n}$. But $|\underline{\nu}| \leq k(n-k)=\operatorname{dim} \mathrm{G}(k, n)$, so we get no quantum contributions except in case of $s_{n}$ with $|\underline{\nu}|=k(n-k)$ and $d=1$. Thus writing $\underline{\hat{c}}=\left(\hat{c}_{1}, \ldots, \hat{c}_{k}\right)$ we obtain $s_{n-k+1}(\underline{\hat{c}})=\ldots=s_{n-1}(\underline{\hat{c}})=0$ in $Q H_{[\omega]}^{*}(\mathrm{G}(k, n))$ and

$$
\begin{aligned}
s_{n}(\underline{\hat{c}}) & =-\hat{c}_{1} s_{n-1}(\underline{\hat{c}})-\ldots-\hat{c}_{k-1} s_{n-k+1}(\underline{\hat{c}})-\hat{c}_{k} s_{n-k}(\underline{\hat{c}}) \\
& =-\hat{c}_{k} s_{n-k}(\underline{\hat{c}})=-\tilde{\Phi}_{[L]}^{0}\left(c_{k}^{\vee}, s_{n-k}^{\vee},[*]\right) \cdot e^{-[\omega](L)},
\end{aligned}
$$

where for the last equality we have used $(* *)$. By Proposition 2.2, to prove Theorem 0.1 , we are left with

Proposition 3.1. $\tilde{\Phi}_{[L]}^{0}\left(c_{k}^{\vee}, s_{n-k}^{\vee},[*]\right)=(-1)^{k}$.

For the proposition we first have to classify holomorphic curves homologous to $[L]=$ $(1, \ldots, k-1, k+1)$.

LEMMA 3.2. Let $C \subset \mathrm{G}(k, n)$ be a (rational) curve homologous to $(1, \ldots, k-1, k+$ $1)$. Then $C$ is a Schubert variety $\Omega_{\underline{V}}(1, \ldots, k-1, k+1)$, i.e. there are linear subspaces $U \subset W \subset \mathbb{C}^{n}, \operatorname{dim} U=k-1, \operatorname{dim} \bar{W}=k+1$, with $C=\{\Lambda \in \mathrm{G}(k, n) \mid U \subset \Lambda \subset W\}$.

Proof. Since $s_{1}[C]=1$ by the duality theorem, $\operatorname{deg} \iota(C)=1$, so the image of $C$ under the Plücker embedding $\iota: \mathrm{G}(k, n) \rightarrow \mathbb{P}\left(\Lambda^{k} \mathbb{C}^{n}\right)$ is a linear $\mathbb{P}^{1}$. The image of $\mathrm{G}(k, n)$ consists precisely of (rays of) decomposable vectors $v_{1} \wedge \ldots \wedge v_{k}=\iota\left(\left\langle v_{1}, \ldots, v_{k}\right\rangle\right) \in$ $\Lambda^{k} \mathbb{C}^{n}$. Locally, the vectors $v_{1}, \ldots, v_{k}$ may be chosen to vary smoothly with $\Lambda \in$ $\mathrm{G}(k, n)$ : In fact, the standard (affine) coordinate neighbourhood of $\Lambda \in \mathrm{G}(k, n)$ is 
$\operatorname{Hom}\left(\Lambda, \mathbb{C}^{n} / \Lambda\right)$ with $\Psi: \operatorname{Hom}\left(\Lambda, \mathbb{C}^{n} / \Lambda\right) \rightarrow \mathrm{G}(k, n), \varphi \mapsto\langle v+\varphi(v) \mid v \in \Lambda\rangle$ (in particular $0 \in \operatorname{Hom}\left(\Lambda, \mathbb{C}^{n} / \Lambda\right)$ corresponds to $\left.\Lambda\right)$. Now fixing a basis $v_{1}, \ldots, v_{k}$ of $\Lambda, \iota \circ \Psi$ may be represented (lifted to $\Lambda^{k} \mathbb{C}^{n}$ ) by

$$
\varphi \mapsto\left(v_{1}+\varphi\left(v_{1}\right)\right) \wedge \ldots \wedge\left(v_{k}+\varphi\left(v_{k}\right)\right) .
$$

Thus choosing $\Lambda^{\prime} \in C$ sufficiently close to $\Lambda \in C$ and letting $e_{1}, \ldots, e_{l}$ be a basis of $\Lambda \cap \Lambda^{\prime}$, completed by $e_{l+1}, \ldots, e_{k}$ and $e_{l+1}^{\prime}, \ldots, e_{k}^{\prime}$ to a basis of $\Lambda$ and $\Lambda^{\prime}$ respectively, we have for $t \in \mathbb{C}$ small

$$
e_{1} \wedge \ldots \wedge e_{k}+t \cdot e_{1} \wedge \ldots \wedge e_{l} \wedge e_{l+1}^{\prime} \wedge \ldots \wedge e_{k}^{\prime}=v_{1}(t) \wedge \ldots \wedge v_{k}(t)
$$

with $v_{i}(0)=e_{i}$ by construction. Taking $\left.\frac{d}{d t}\right|_{t=0}$ yields

$$
e_{1} \wedge \ldots \wedge e_{l} \wedge e_{l+1}^{\prime} \wedge \ldots \wedge e_{k}^{\prime}=\dot{v}_{1}(0) \wedge e_{2} \wedge \ldots \wedge e_{k}+\ldots+e_{1} \wedge \ldots \wedge e_{k-1} \wedge \dot{v}_{k}(0)
$$

As one sees by expanding $\dot{v}_{i}(0)$ in terms of a basis of $\mathbb{C}^{n}$ containing $\left\{e_{1}, \ldots, e_{k}\right.$, $\left.e_{l+1}^{\prime}, \ldots, e_{k}^{\prime}\right\}$ we may gather the linearly independent terms with and without $e_{k}$ to form two equations. The left-hand side of the equation above belongs to the latter $\left(\Lambda \neq \Lambda^{\prime} \Rightarrow l<k\right)$, so we get

$$
e_{1} \wedge \ldots \wedge e_{l} \wedge e_{l+1}^{\prime} \wedge \ldots \wedge e_{k}^{\prime}=e_{1} \wedge \ldots \wedge e_{k-1} \wedge\left(\dot{v}_{k}(0)-\lambda \cdot e_{k}\right)
$$

where $\lambda \in \mathbb{C}$ is chosen in such a way that $\dot{v}_{k}(0)-\lambda \cdot e_{k}$ lies in the span of the basis vectors different from $e_{k}$. By linear independence of wedge products of a basis of $\mathbb{C}^{n}$ this shows $l=k-1$ and $e_{k}^{\prime}=\dot{v}_{k}(0)-\lambda e_{k}$. In view of the linearity of $\iota(C)$ we conclude

$$
\iota(C)=\left\{\left[t \cdot e_{1} \wedge \ldots \wedge e_{k-1} \wedge e_{k}^{\prime}+u \cdot e_{1} \wedge \ldots \wedge e_{k-1} \wedge e_{k}\right] \mid[t: u] \in \mathbb{P}^{1}\right\},
$$

so $C$ is the Schubert variety $\Omega_{\underline{V}}(1, \ldots, k-1, k+1)$ belonging to a flag $\underline{V}$ with $V_{k-1}=$ $\left\langle e_{1}, \ldots, e_{k-1}\right\rangle=: U$ and $V_{k+1}=\left\langle e_{1}, \ldots, e_{k-1}, e_{k}, e_{k}^{\prime}\right\rangle=: W$.

LEMMA 3.3. Let $A_{1}=\Omega_{V^{1}}(n-k, n-k+1, \ldots, n-1)=\{1, \ldots, 1\}^{\vee}=(-1)^{k} c_{k}^{\vee}$, $A_{2}=\Omega_{\underline{V}^{2}}(1, n-k+2, \ldots, n)=\{n-k, 0, \ldots, 0\}^{\vee}=s_{n-k}^{\vee}, A_{3}=\{*\}=\Omega_{\underline{V}^{3}}(1, \ldots, k)=$ $\{n-k, \ldots, n-k\}^{\vee}$, where $\underline{V}^{1}, \underline{V}^{2}, \underline{V}^{3}$ are three transversal flags, i.e. $\operatorname{dim} V_{i}^{1} \cap V_{j}^{2} \cap$ $V_{k}^{3}=\max \{0, i+j+k-2 n\}$ for any $i, j, k$.

Then there is exactly one rational curve $C$ homologous to $[L]$ and with $C \cap A_{i} \neq \emptyset$, $i=1,2,3$. Moreover, $C \cdot A_{1}=C \cdot A_{2}=C \cdot A_{3}=1$.

The lemma can be proved by doing intersection theory on the flag manifold $\mathrm{F}(k-$ $1, k+1 ; n)$ which parametrizes rational curves of minimal degree by the preceding lemma, and using the two obvious maps $\pi: \mathrm{F}(k-1, k+1 ; n) \rightarrow \mathrm{G}(k, n)$ and $p$ : $\mathrm{F}(k-1, k+1 ; n) \rightarrow \mathrm{G}(k, n)$ (calculate $\left.\left(p_{*} \pi^{*}\left[A_{1}\right]\right) \cdot\left(p_{*} \pi^{*}\left[A_{2}\right]\right) \cdot\left(p_{*} \pi^{*}\left[A_{3}\right]\right)\right)$. This method might be appropriate for more general $A_{1}, A_{2}, A_{3}$, yet in our case an explicit linear algebra argument is simpler and even more enlightening.

Proof. We need to find three $k$-planes $\Lambda^{1}, \Lambda^{2}, \Lambda^{3}$ and subspaces $U, W \subset \mathbb{C}^{n}$, $\operatorname{dim} U=k-1, \operatorname{dim} W=k+1$ with

1. $U \subset \Lambda^{1} \cap \Lambda^{2} \cap \Lambda^{3} \subset \Lambda^{1}+\Lambda^{2}+\Lambda^{3} \subset W$.

2. $\operatorname{dim} \Lambda^{1} \cap V_{n-k+(i-1)}^{1} \geq i, i=1, \ldots, k$.

3. $V_{1}^{2} \subset \Lambda^{2}$.

4. $\Lambda^{3}=V_{k}^{3}$, 
(1) says that $\Lambda^{1}, \Lambda^{2}, \Lambda^{3}$ lie on the rational curve $C$ defined by $U$ and $W$ according to Lemma 3.2, whereas (2)-(4) rephrase the conditions $\Lambda_{i}=A_{i} \cap C, i=1,2,3$. We now use transversality of the flags $\underline{V}^{1}, \underline{V}^{2}, \underline{V}^{3}$ : From (3), (4) and (1) we readily deduce $W=V_{k}^{3}+V_{1}^{2}$, and (2) with $i=k$ shows $\Lambda^{1} \subset V_{n-1}^{1}$, so by (4) and (1) we get $U=V_{k}^{3} \cap V_{n-1}^{1}$. This choice of $U, W$ implies $\Lambda^{1}=W \cap V_{n-1}^{1}, \Lambda^{2}=U+V_{1}^{2}, \Lambda^{3}=V_{k}^{3}$. Conversely, these $\Lambda^{1}, \Lambda^{2}, \Lambda^{3}$ fulfill (1)-(4).

Proof of Proposition 3.1. Let $I$ be the integrable complex structure on $\mathrm{G}(k, n)$. Consider the evaluation map

$$
\text { ev }: \Sigma \times \mathcal{M}_{[L]}\left(\mathbb{P}^{1}, I, 0\right) \longrightarrow \mathrm{G}(k, n) \times \mathrm{G}(k, n) \times \mathrm{G}(k, n) .
$$

Since the tangent bundle of $G(k, n)$ is generated by global sections, so is $N_{L / G(k, n)}$ for any line $L$. Hence $H^{1}\left(N_{L / \mathrm{G}(k, n)}=0\right.$ for any such line and $\mathcal{M}_{[L]}\left(\mathbb{P}^{1}, I, 0\right)=$ $\operatorname{Hom}_{[L]}\left(\mathbb{P}^{1}, M\right)$ is a manifold of expected dimension $n+k(n-k)$ (in fact a PGL(2)bundle over $F(k-1, k+1 ; n))$. By the preceding lemma the preimage under ev of $A_{1} \times A_{2} \times A_{3}$ for any $A_{i}$ belonging to transversal flags consists of exactly one $f \in \mathcal{M}_{[L]}\left(\mathbb{P}^{1}, I, 0\right)$. But letting the transversal flags vary, the variation of ev $(f) \cap$ $A_{1} \times A_{2} \times A_{3}$ sweeps out the normal directions of $A_{1} \times A_{2} \times A_{3}$ in $\mathrm{G}(k, n)^{3}$ as follows explicitely from the proof of Lemma 3.3.

This finishes the proof of Theorem 0.1 .

REMARK 3.4. (ON ALGEBRAICITY) While we use the symplectic definition of GW-invariants here, our arguments are essentially algebraic. In fact, the computation of the invariant $\tilde{\Phi}_{[L]}^{0}\left(c_{k}^{\vee}, s_{n-k}^{\vee},[*]\right)$ is done by ordinary intersection theory on a moduli space of algebraic lines in $\mathrm{G}(k, n)$. And in a joint work of the second named author with $\mathrm{J}$. Li it is shown by purely algebraic methods that for homogeneous varieties the "naive", algebraic, genus zero invariants, defined by intersection theory on algebraic moduli spaces, also obey associativity [LiTi] (and coincide with the symplectic GWinvariants). So these symplectic GW-invariants are "enumerative" in the sense that they count numbers of actual holomorphic curves.

For the Grassmannians analogous results have been given independently by [Be1]. In this paper a different compactification of $\operatorname{Hom}(\Sigma, \mathrm{G}(k, n))$ is used (by a quot scheme). The author also shows enumerativeness of the invariants for curves of higher genus and sufficiently high degree, and the induction formula for the genus (Proposition 4.4 below).

More recently, an enumerative theory has been worked out by Behrend and Manin for genus zero invariants and so-called convex projective manifolds by using Kontsevich's very pretty concept of "stable maps" [BeMa]. A smooth variety $M$ is defined to be convex if $H^{1}\left(\mathbb{P}^{1}, \varphi^{*} T_{M}\right)=0$ for any $\varphi \in \operatorname{Hom}\left(\mathbb{P}^{1}, M\right)$. This includes homogeneous varieties for these have globally generated tangent bundles.

In all these examples $\operatorname{Hom}\left(\mathbb{P}^{1}, M\right)$ is smooth of the expected dimension. The boundary being of lower dimension, intersection theory on any compactification (or of the image of the moduli space in $M^{s}$ ) gives the same result.

4. Formulae of Vafa-Intriligator type. The main results of [RuTi] show how to compute higher GW-invariants (i.e. with more than three entries or for higher genus Riemann surfaces) from the genus 0 three-point functions inductively. Namely, for $g>0$

$$
\tilde{\Phi}_{[\omega]}^{g}\left(B_{1}, \ldots, B_{s}\right)=\sum_{i, j} \eta^{i j} \tilde{\Phi}_{[\omega]}^{g-1}\left(B_{1}, \ldots, B_{s}, A_{i}, A_{j}\right)
$$


$\left(\eta_{i j}\right)$ the intersection matrix with respect to a basis $\left\{A_{i}\right\}$ of $H^{*}(M, \mathbb{Q})$. More invariantly, the right-hand side is the trace with respect to $\eta$ of the bilinear form

$$
H^{*}(M, \mathbb{C}) \times H^{*}(M, \mathbb{C}) \longrightarrow \mathbb{C}, \quad\left(B^{\prime}, B^{\prime \prime}\right) \longmapsto \tilde{\Phi}_{[\omega]}^{g-1}\left(B_{1}, \ldots, B_{s}, B^{\prime}, B^{\prime \prime}\right) .
$$

Secondly, for $g=0$ and $1<r<s-1$ (otherwise trivial) we have the composition law

$$
\tilde{\Phi}_{[\omega]}^{0}\left(B_{1}, \ldots, B_{s}\right)=\sum_{i, j} \eta^{i j} \tilde{\Phi}_{[\omega]}^{0}\left(B_{1}, \ldots, B_{r}, A_{i}\right) \cdot \tilde{\Phi}_{[\omega]}^{0}\left(A_{j}, B_{r+1}, \ldots, B_{s}\right),
$$

a trace with respect to $\eta$ as well .For $s=4$ and $r=2$ this equation states the associativity of quantum products. Our goal in this section is to give a closed formula for higher invariants in terms of the relations $f_{1}^{[\omega]}, \ldots, f_{k}^{[\omega]}$ of the quantum cohomology ring.

Putting all $\tilde{\Phi}_{[\omega]}^{g}$ together for different $s$ we get a $\mathbb{C}$-linear map

$$
\langle\rangle_{g}: \mathbb{C}\left\langle X_{1}, \ldots X_{n}\right\rangle \longrightarrow \mathbb{C}, \quad X_{1}^{\nu_{1}} \ldots X_{n}^{\nu_{n}} \longmapsto \tilde{\Phi}_{[\omega]}^{g}\left(X_{1}^{\vee}, \ldots, X_{1}^{\vee}, \ldots, X_{n}^{\vee}, \ldots, X_{n}^{\vee}\right),
$$

the Poincaré-dual $X_{i}^{\vee}$ of $X_{i}$ occurring $\nu_{i}$-times. But from the composition law, letting $\alpha_{i}$ denote the Poincaré-dual of $A_{i}$,

$$
\hat{X}_{1}^{\nu_{1}} \wedge_{\mathrm{Q}} \ldots \wedge_{\mathrm{Q}} \hat{X}_{n}^{\nu_{n}}=\sum_{i j} \eta^{i j} \tilde{\Phi}_{[\omega]}^{0}\left(X_{1}^{\vee}, \ldots, X_{1}^{\vee}, \ldots, X_{n}^{\vee}, A_{i}\right) \hat{\alpha}_{j}
$$

so $\left\langle X_{1}^{\nu_{1}} \ldots X_{n}^{\nu_{n}}\right\rangle_{0}=\tilde{\Phi}_{[\omega]}^{0}\left(X_{1}^{\vee}, \ldots, X_{1}^{\vee}, \ldots, X_{n}^{\vee},[M]\right)$ is nothing but the coefficient of the class $[\Omega]$ of the normalized volume form in $\hat{X}_{1}^{\nu_{1}} \wedge_{Q} \ldots \wedge_{\mathrm{Q}} \hat{X}_{n}^{\nu_{n}}$. That is, \langle\rangle$_{0}$ factorizes in the following way:

$$
\mathbb{C}\left\langle X_{1}, \ldots, X_{n}\right\rangle \longrightarrow \mathbb{C}\left\langle X_{1}, \ldots, X_{n}\right\rangle /\left(f_{1}^{[\omega]}, \ldots, f_{k}^{[\omega]}\right) \simeq H_{[\omega]}^{*}(M) \stackrel{\text { top }}{\longrightarrow} H^{2 n}(M, \mathbb{C})
$$

when $H^{2 n}(M, \mathbb{C})$ is identified with $\mathbb{C}$ by sending $[\Omega]$ to 1 . In case $n=k$ and the generators have even degree, i.e. $H^{*}(M, \mathbb{C})$ is a (commutative) complete intersection ring, one can use higher dimensional residues to express this map more explicitely (we refer the reader to $[\mathrm{GrHa}]$ and $\left[\mathrm{T}_{\mathrm{S}}\right]$ for the general facts on residues to be used):

Recall that the residue in $a \in \mathbb{C}$ of $F \in \mathbb{C}\left[X_{1}, \ldots, X_{k}\right]$ with respect to a polynomial mapping $g=\left(g_{1}, \ldots, g_{k}\right): \mathbb{C}^{k} \rightarrow \mathbb{C}^{k}$ with $g^{-1}(0)$ finite is defined by

$$
\operatorname{res}_{g}(a ; F):=\frac{1}{(2 \pi i)^{k}} \int_{\Gamma_{a}^{\varepsilon}} \frac{F}{g_{1} \cdots g_{k}} d X_{1} \ldots d X_{k}
$$

with $\Gamma_{a}^{\varepsilon}=\left\{x \in U(a)|| g_{i}(x) \mid=\varepsilon\right\}, U(a)$ a neighbourhood of $a$ with $g^{-1}(0) \cap$ $U(a)=\{a\}$ and $\varepsilon$ so small that $\Gamma_{a}^{\varepsilon}$ lies relatively compact in $U(a) . \Gamma_{a}^{\varepsilon}$ is smooth for almost all $\varepsilon$ by Sard's Theorem and has a canonical orientation by the $k$-form $d\left(\arg g_{1}\right) \wedge \ldots \wedge d\left(\arg g_{k}\right) \mid \Gamma_{a}^{\varepsilon}$. (This local residue of course makes sense for holomorphic $g$ and $F \in \mathcal{O}_{a}$, but the polynomial case, to which the general case may easily be reduced, is sufficient for our purposes). Note that the hypothesis og $g^{-1}(0)$ being finite is equivalent to the saying that the ring $\mathbb{C}\left[X_{1}, \ldots, X_{k}\right] /\left(g_{1}, \ldots, g_{k}\right)$ is Artinian, i.e. finite dimensional as vector space over $\mathbb{C}$. We define the total residue

$$
\operatorname{Res}_{g}(F):=\sum_{a \in g^{-1}(0)} \operatorname{res}_{g}(a ; F)
$$


which is also known as Grothendieck residue symbol $\left(\begin{array}{c}F \\ g_{1}, \ldots, g_{k}\end{array}\right)$ in the context of duality theory in algebraic geometry [Ha]. Let $J=\operatorname{det}\left(\frac{\partial g_{i}}{\partial X_{j}}\right)$ be the Jacobian of $g$. For regular values $y$ of $g$

$$
\operatorname{Res}_{g-y}(F)=\sum_{x \in g^{-1}(y)}\left(\frac{F}{J}\right)(x)=: \operatorname{tr}\left(\frac{F}{J}\right)(0) .
$$

Therefore $\operatorname{tr}(F / J)$ extends holomorphically to a neighbourhood of 0 (the extension will be denoted $\operatorname{tr}(F / J)$ as well $)$ and

$$
\operatorname{Res}_{g}(F)=\operatorname{tr}\left(\frac{F}{J}\right)(0) .
$$

One abstract feature in our setting is that we have weights $d_{i}$ associated to $X_{i}$ and that our relations $f_{i}^{[\omega]}$ form a Gröbner basis of the relation ideal with respect to these weights, i.e.

$$
\left(\operatorname{In} f_{1}^{[\omega]}, \ldots, \operatorname{In} f_{k}^{[\omega]}\right)=\left(f_{1}, \ldots, f_{k}\right)=\operatorname{In}\left(f_{1}^{[\omega]}, \ldots, f_{k}^{[\omega]}\right),
$$

where "In" means taking initial forms with respect to the weight order. This is trivial in our case since we started with the homogeneous generators $f_{1}, \ldots, f_{k}$ of the relation ideal in a presentation of $H^{*}(M, \mathbb{C})$. In such situations one can describe the residue map algebraically as follows:

Proposition 4.1. Let $R=\mathbb{C}\left[X_{1}, \ldots, X_{k}\right] /\left(g_{1}, \ldots, g_{k}\right)$ be Artinian with $\left\{g_{i}\right\}$ a Gröbner basis of the relation ideal with respect to weights $d_{i}$ of $X_{i}$. Put $N:=$ $\sum_{i} \operatorname{deg} g_{i}-\sum_{i} d_{i}, R_{<N}:=\left\{F \in \mathbb{C}\left[X_{1}, \ldots, X_{k}\right] \mid \operatorname{deg} F<N\right\} /\left(g_{1}, \ldots, g_{k}\right)$ and $J=$ $\operatorname{det}\left(\frac{\partial g_{i}}{\partial X_{j}}\right)$. Then

$$
R=R_{<N} \oplus \mathbb{C} \cdot J
$$

and the total residue map $\operatorname{Res}_{g}: \mathbb{C}\left[X_{1}, \ldots, X_{k}\right] \rightarrow \mathbb{C}$ factorizes via the projection onto the second factor as follows

$$
\mathbb{C}\left[X_{1}, \ldots, X_{k}\right] \stackrel{\text { can }}{\longrightarrow} R \stackrel{\mathrm{pr}_{2}}{\longrightarrow} \mathbb{C} \cdot J \longrightarrow \mathbb{C},
$$

where the last map sends $J$ to $\operatorname{dim}_{\mathbb{C}} R$.

Proof. To check the normalization we observe

$$
\operatorname{Res}_{g}(J)=\left(\operatorname{tr}_{g} 1\right)(0)=\text { degree of } g \text { over } 0=\operatorname{dim}_{\mathbb{C}} R .
$$

The last equality is generally true by flatness if the covering space is Cohen-Macaulay, cf. eg. [Fi]. Next, it is well known that the residue vanishes on elements of $\left(g_{1}, \ldots, g_{k}\right)$. The claim thus reduces to $\operatorname{ker}\left(\operatorname{Res}_{g}\right) /\left(g_{1}, \ldots, g_{k}\right)=R_{<N}$.

If $\operatorname{deg} F<N$ then $F d X_{1} \ldots d X_{k} / g_{1} \cdots g_{k}$ extends to a rational differential form $\varphi$ on the weighted projective space $V=\mathbb{P}_{\left(1, d_{1}, \ldots, d_{k}\right)}$ with polar divisor $D_{1}+\ldots+D_{k} \in$ $\operatorname{Div}(V), D_{i}$ the natural extension of the divisor $\left(g_{i}\right)$ to $V$. The point of course is that the divisor at infinity $V \backslash \mathbb{C}^{k}$ is not a polar divisor of $\varphi$. We claim $\left|D_{1}\right| \cap \ldots \cap\left|D_{k}\right| \subset$ $\mathbb{C}^{k}$. In fact, the restriction of the homogenization of $g_{i}$ to $V \backslash \mathbb{C}^{k} \simeq \mathbb{P}_{\left(d_{1}, \ldots, d_{k}\right)}$ is just $\operatorname{In} g_{i}$ and $V\left(\operatorname{In} g_{1}, \ldots, \operatorname{In} g_{k}\right)=\{0\} \in \mathbb{C}^{k}$. The latter follows because otherwise $\operatorname{dim} \operatorname{Spec} \mathbb{C}\left[X_{1}, \ldots, X_{k}\right] /\left(\operatorname{In} g_{1}, \ldots, \operatorname{In} g_{k}\right)>0$ by homogeneity. But from the Gröbner basis property we have

$$
\operatorname{dim}_{\mathbb{C}} \mathbb{C}\left[X_{1}, \ldots, X_{k}\right] /\left(\operatorname{In} g_{1}, \ldots, \operatorname{In} g_{k}\right)=\operatorname{dim}_{\mathbb{C}} \mathbb{C}\left[X_{1}, \ldots, X_{k}\right] /\left(g_{1}, \ldots, g_{k}\right) .
$$


We may thus desingularize $V$ (at infinity) without violating the discreteness of $\left|D_{1}\right| \cap$ $\ldots \cap\left|D_{k}\right|$. We keep the notations for the pulled-back objects. Now the global residue theorem tells that on the compact manifold $V$ the sum of the local residues of $\varphi$ with respect to $D_{1}, \ldots, D_{k}$ equals zero (the local residue $\operatorname{res}_{g}(a ; F)$ makes sense on manifolds by noting that it depends only on the associated rational differential form $F d X_{1} \ldots d X_{k} / g_{1} \ldots g_{k}$ and the divisors $\left.\left(g_{1}\right), \ldots,\left(g_{k}\right)\right)$. This proves $\operatorname{Res}_{g}(F)=0$ in case $\operatorname{deg} F<N$.

The second case is $F$ homogeneous of degree $>N$. We show $F \in\left(\operatorname{In} g_{1}, \ldots, \operatorname{In} g_{k}\right)$. This is an easy generalization of a theorem of Macaulay (cf. e.g. [Ts]) to the weighted situation. Namely, let $Q$ be any homogeneous polynomial and set $P=F \cdot G$. With $Q:=\operatorname{In}\left(g_{1}\right) \cdots \operatorname{In}\left(g_{k}\right)$ we have

$$
\frac{P}{Q} d X_{1} \ldots d X_{k}=\left(\operatorname{deg} P-\operatorname{deg} Q+\sum_{i} d_{i}\right)^{-1} d \sigma=(\operatorname{deg} P-N)^{-1} d \sigma
$$

with

$$
\sigma=\frac{P}{Q} \sum_{j=1}^{k}(-1)^{j-1} d_{j} \cdot X_{j} d X_{1} \ldots \widehat{d X_{j}} \ldots d X_{k}
$$

where - means that this entry is to be left out. This is a simple check using the weighted Euler formula $\sum_{j} d_{j} X_{j} \frac{\partial H}{\partial X_{j}}=\operatorname{deg}(H) \cdot P, H$ weighted homogeneous (same proof as usual). Thus

$$
\operatorname{Res}_{\operatorname{In} g}(F \cdot G)=\sum_{a} \int_{\Gamma_{a}^{\varepsilon}} d \sigma=0
$$

for all (homogeneous) $G \in \mathbb{C}\left[X_{1}, \ldots, X_{k}\right]$. But this implies $F \in\left(\operatorname{In} g_{1}, \ldots, \operatorname{In} g_{k}\right)$ by the "duality theorem", cf. [Ts] (this is Poincaré duality in the case of cohomology rings). Modulo $\left(g_{1}, \ldots, g_{k}\right)$ this means that we may reduce $F$ to lower degree. So proceeding by induction $N$ turns out to be "top-degree" in $R$ in that all elements of $R$ can be represented by polynomials of degree $\leq N$.

What remains to be checked is that for $F$ homogeneous of degree $N$, either $\operatorname{Res}_{g}(F) \neq 0$ or $F /\left(g_{1}, \ldots, g_{k}\right) \in R_{<N}$. In fact, if $\operatorname{Res}_{g}(F)=0$ then $\operatorname{Res}_{\operatorname{In}(g)}(F \cdot G)=$ $0 \forall G \in \mathbb{C}\left[X_{1}, \ldots, X_{k}\right]$, because for $G$ homogeneous of positive degree this has just been shown. So again $F \in\left(\operatorname{In} g_{1}, \ldots, \operatorname{In} g_{k}\right)$, i.e. modulo $\left(g_{1}, \ldots, g_{k}\right), F$ may be represented by a polynomial of degree $<N$.

REMARK 4.2. The decomposition $R=R_{<N} \oplus\langle J\rangle$ is not canonical but rather depends on the particular presentation of $R$. This may be seen either in elementary terms from the transformation formula for residues or as manifestation of the choice of an isomorphism $\operatorname{Ext}_{V}^{k}\left(\mathcal{O}_{Z}, \Omega_{V}^{k}\right) \simeq H^{0}\left(V, \mathcal{O}_{Z}\right)$ in the duality morphism

$$
\operatorname{Ext}_{V}^{k}\left(\mathcal{O}_{Z}, \Omega_{V}^{k}\right) \times H^{0}\left(V, \mathcal{O}_{Z}\right) \rightarrow \mathbb{C}
$$

induced by the global residue. For quantum cohomology rings and weightings coming from cohomology, however, $R_{<N}=\oplus_{d<N} H^{d}(M, \mathbb{C}), N=\operatorname{dim}_{\mathbb{C}} M$ and $\langle Y\rangle=H^{2 N}(M, \mathbb{C})$, so the decomposition has an invariant meaning in this case.

In the quantum cohomology ring the top-degree class $\mathbb{C} \cdot J$ is thus spanned by the class $[\Omega]$ of the volume form. Let $F_{[\Omega]}$ be a polynomial of degree $N$ representing $[\Omega]$ (modulo $\left(f_{i}^{[\omega]}\right)$ or modulo $\left(f_{i}\right)$, this will yield the same result), and 
set $c=1 / \operatorname{Res}_{f}{ }^{[\omega]}\left(F_{[\Omega]}\right)$. By the interpretation of $\langle F\rangle_{0}$ as coefficient of $[\Omega]$ of $F\left(\hat{X}_{1}, \ldots, \hat{X}_{k}\right) \in Q H_{[\omega]}^{*}(M)$ we conclude (for $H^{*}(M, \mathbb{C})$ a complete intersection)

$$
\langle F\rangle_{0}=c \cdot \operatorname{Res}_{f[\omega]}(F)=c \cdot \operatorname{tr}_{f[\omega]}\left(\frac{F}{J}\right)(0) .
$$

To incorporate the higher genus case we prove

Lemma 4.3. For notations as in Proposition 4.1 and $F \in \mathbb{C}\left[X_{1}, \ldots, X_{k}\right]$ let $B_{F}$ be the bilinear form $R \times R \rightarrow \mathbb{C},(\alpha, \beta) \mapsto \operatorname{Res}_{g}\left(F \cdot G_{\alpha} \cdot G_{\beta}\right)\left(\right.$ with $G_{\alpha}, G_{\beta} \in \mathbb{C}\left[X_{1}, \ldots, X_{k}\right]$ representing $\alpha, \beta$ - this is well-defined), $\eta=B_{1}$ the "intersection form". Then

$$
\operatorname{tr}_{\eta} B_{F}=\operatorname{Res}_{g}(F \cdot J)
$$

Proof. We give a basis-free, geometric proof. By definition $\operatorname{tr}_{\eta} B_{F}$ is the trace of the endomorphism $\mu_{F}: R \rightarrow R$ of multiplication by (the class of) $F$. Putting $Z=\operatorname{Spec} R$ we have $R=\oplus_{z \in g^{-1}(0)} \mathcal{O}_{Z, z}$. For $z \in g^{-1}(0), F-F(z)$ is nilpotent in $\mathcal{O}_{Z, z}$ (for it has value 0 in $z$ ), so $\left.\mu_{F-F(z)}\right|_{\mathcal{O}_{z, z}}$ has trace 0 and

$$
\operatorname{tr}\left(\mu_{F} \mid \mathcal{O}_{Z, z}\right)=\operatorname{tr}\left(\mu_{F-F(z)} \mid \mathcal{O}_{Z, z}\right)+\operatorname{tr}\left(\mu_{F(z)} \mid \mathcal{O}_{Z, z}\right)=F(z) \cdot \operatorname{dim}_{\mathbb{C}} \mathcal{O}_{Z, z}
$$

Furthermore, $\operatorname{dim}_{\mathbb{C}} \mathcal{O}_{Z, z}=\operatorname{deg}_{z}(g)$, the local mapping degree of $g$ at $z$, so summing up we get

$$
\operatorname{tr}_{\eta} B_{F}=\sum_{z \in g^{-1}(0)} \operatorname{deg}_{z}(g) \cdot F(z)=\operatorname{tr}_{g}(F)
$$

which is nothing but $\operatorname{Res}_{g}(F \cdot J)$ as claimed.

In view of the reduction formula to lower genus stated above we conclude a mathematical version of Witten's "handle gluing formula" in topological QFT [Wi2].

Proposition 4.4. Let $H^{*}(M, \mathbb{Q})=\mathbb{Q}\left[X_{1}, \ldots, X_{k}\right] /\left(f_{1}, \ldots, f_{k}\right)$ (the commutative complete intersection case) and $f_{i}^{[\omega]}$ the induced relations in $Q H_{[\omega]}^{*}(M)$ as in Proposition 2.2. Put $J=\operatorname{det}\left(\frac{\partial f_{i}^{[\omega]}}{\partial X_{j}}\right)$. Then

$$
\langle F\rangle_{g}=\langle J \cdot F\rangle_{g-1}
$$

holds for all $F \in \mathbb{C}\left[X_{1}, \ldots, X_{k}\right]$.

In other words, multiplication by $J$ acts as "attaching a handle" to our Riemann surface.

We summarize our considerations in the following main result of the present chapter:

THEOREM 4.5. Assumptions as in the preceding proposition then for all $F \in$ $\mathbb{C}\left[X_{1}, \ldots, X_{k}\right]$ the following holds

$$
\langle F\rangle_{g}=c \cdot \operatorname{Res}_{f^{[\omega]}}\left(J^{g} \cdot F\right)=c \cdot \lim _{\substack{y \rightarrow 0 \\ y \text { regular } \\ \text { value of } f^{[\omega]}}} \operatorname{tr}_{f^{[\omega]}}\left(J^{g-1} \cdot F\right)(y),
$$

with $c=1 / \operatorname{Res}_{f[\omega]}\left(F_{[\Omega]}\right), F_{[\Omega]}$ a polynomial representing the class $[\Omega] \in Q H_{[\omega]}^{*}(M)$ of the normalized volume form. 
We emphasize that for $g>0$ or $0 \in \mathbb{C}^{k}$ a regular value of $f^{[\omega]}$ the right-hand side has the form $\sum_{\nu} a_{\nu} F\left(y_{\nu}\right)$ with $g^{-1}(0)=\left\{y_{\nu}\right\}$ and constants $a_{\nu}$ independent of $F$ !

Note also that 0 is a regular value of $f^{[\omega]}$ iff $\operatorname{dim}_{\mathbb{C}} \mathcal{O}_{Z, z}=1$ for all $z \in\left(f^{[\omega]}\right)^{-1}(0)$, $Z=\operatorname{Spec} \mathbb{C}\left[X_{1}, \ldots, X_{k}\right] /\left(f_{1}^{[\omega]}, \ldots, f_{k}^{[\omega]}\right)$, which is if and only if

$$
\operatorname{dim}_{\mathbb{C}} H^{*}(M, \mathbb{C})=\sharp\left(f^{[\omega]}\right)^{-1}(0)
$$

(" $\geq "$ always).

We claim that this is the case for $\mathrm{G}(k, n)$ : We have a basis of Schubert classes $\left\{\lambda_{1}, \ldots, \lambda_{k}\right\}$ parametrized by sequences $n-k \geq \lambda_{1} \geq \ldots \geq \lambda_{k} \geq 0$. The latter are in $1-1$ correspondence with subsets $\left\{\lambda_{k}+1, \lambda_{k-1}+2, \ldots, \lambda_{1}+k\right\}$ of $\{1, \ldots, n\}$, so $\operatorname{dim}_{\mathbb{C}} H^{*}(\mathrm{G}(k, n), \mathbb{C})=\left(\begin{array}{l}n \\ k\end{array}\right)$.

To find $\left(\begin{array}{l}n \\ k\end{array}\right)$ distinct elements in $\left(f^{[\omega]}\right)^{-1}(0)$, we use a description of the cohomology ring coming from the study of the corresponding Landau-Ginzburg model in physics [Va1]: There is a polynomial $W$ in the $X_{i}$ ("Landau-Ginzburg potential") so that in the notations of Theorem 0.1

$$
Y_{n+1-i}\left(X_{1}, \ldots, X_{k}\right)=\frac{\partial W}{\partial X_{i}}
$$

and then $f_{i}^{[\omega]}=\frac{\partial W^{[\omega]}}{\partial X_{i}}$ with $W^{[\omega]}:=W+(-1)^{k} e^{-[\omega](L)} \cdot X_{1}$. W has a simple description in terms of Chern roots, i.e. after composition with the $k$ !-fold branched covering $\Sigma: \mathbb{C}^{k} \longrightarrow \mathbb{C}^{k}$

$$
\Sigma(\underline{\lambda})=\left(-\sigma_{1}(\underline{\lambda}), \sigma_{2}(\underline{\lambda}) \ldots,(-1)^{k} \sigma_{k}(\underline{\lambda})\right),
$$

$\sigma_{i}$ the elementary symmetric polynomials:

$$
W \circ \Sigma=-\frac{1}{n+1} \sum_{i} \lambda_{i}^{n+1}
$$

Thus

$$
W^{[\omega]} \circ \Sigma=-\sum_{i=1}^{k}\left(\frac{\lambda_{i}^{n+1}}{n+1}-(-1)^{k} e^{-[\omega](L)} \cdot \lambda_{i}\right) .
$$

This all is an easy formal consequence of the algebraic relations between Chern and Segre classes on one side and Chern roots on the other, cf. [BDW] for a mathematical account.

Now in a non-degenerate value $\Sigma(\underline{\lambda})$ of $\Sigma, W^{[\omega]}$ is degenerate iff

$$
\frac{\left(\partial W^{[\omega]} \circ \Sigma\right)}{\partial \lambda_{i}}(\underline{\lambda})=0
$$

for all $i$. Non-degeneracy of $\Sigma$ in $\underline{\lambda}$ is equivalent to $\lambda_{i} \neq \lambda_{j}$ for $i \neq j$. Solving the equation means

$$
\lambda_{i}^{n}=(-1)^{k} e^{-[\omega](L)}, \quad i=1, \ldots, k
$$


This system of equations has exactly $n \cdot(n-1) \cdots(n-k+1)$ solutions with pairwise distinct $\lambda_{i}$. The fiber of $\Sigma$ over the regular value $\Sigma(\underline{\lambda})$ consists of the $k$ ! permutations of $\left\{\lambda_{1}, \ldots, \lambda_{k}\right\}$, so we get precisely $\left(\begin{array}{l}n \\ k\end{array}\right)$ distinct elements of $\left(f^{[\omega]}\right)^{-1}(0)$, as wanted. Applying the last theorem we obtain

THEOREM 4.6. (Formula of Vafa and Intriligator) (Notations as in Theorem 0.1) For any $[\omega] \in H^{1,1}(\mathrm{G}(k, n))$ the set $C^{[\omega]}$ of critical points of $W^{[\omega]}$ is finite and all of these are non-degenerate. Moreover, for any $F \in \mathbb{C}\left[X_{1}, \ldots, X_{k}\right]$ the following formula for the genus $g$ GW-invariants of $\mathrm{G}(k, n)$ holds:

$$
\langle F\rangle_{g}^{[\omega]}=(-1)^{n+k(k-1) / 2} \sum_{\underline{x} \in C[\omega]} \operatorname{det}\left(\frac{\partial^{2} W^{[\omega]}}{\partial X_{i} \partial X_{j}}\right)^{g-1}(\underline{x}) \cdot F(\underline{x}) .
$$

Proof. What is left is a check of the normalization. This amounts to calculate the residue of $[\Omega]=(-1)^{n-k} X_{k}^{n-k}$ with respect to $f^{[\omega]}$. We set $a=(-1)^{k} e^{[\omega](L)}$. Using standard properties of residues one gets

$$
\operatorname{Res}_{f[\omega]}\left((-1)^{n-k} X_{k}^{n-k}\right)=\frac{(-1)^{n}}{k !} \operatorname{Res}_{\underline{\lambda}^{n}-\underline{a}}\left(\prod_{i=1}^{k} \lambda_{i}^{n-k} \prod_{i<j}\left(\lambda_{i}-\lambda_{j}\right)^{2}\right)
$$

where

$$
\underline{\lambda}^{n}-\underline{a}=\left(\lambda_{1}^{n}-a, \ldots, \lambda_{k}^{n}-a\right)=-\frac{\partial\left(W^{[\omega]} \circ \Sigma\right)}{\partial \lambda_{i}}=-f^{[\omega]} \circ \frac{\partial \Sigma}{\partial \lambda_{i}} .
$$

In fact, $X_{k}^{n-k} \circ \Sigma(\underline{\lambda})=\left(\prod \lambda_{i}\right)^{n-k}, \prod_{i<j}\left(\lambda_{i}-\lambda_{j}\right)^{2}$ is the squared Jacobian of $\Sigma$ and $k$ ! is the degree of $\Sigma$. Since terms of degree less than $k(n-1)$ modulo $\left(\lambda_{i}^{n}-a\right)$ have vanishing residue (Proposition 4.1) only the term $\prod_{i=1}^{k} \lambda_{i}^{k-1}$ from the expansion of $\prod_{i<j}\left(\lambda_{i}-\lambda_{j}\right)^{2}$ contributes. The coefficient of this term is $(-1)^{k(k-1) / 2} \cdot k !$ as one sees by writing $\prod_{i<j}\left(\lambda_{i}-\lambda_{j}\right)^{2}$ as determinant of a product of a Vandermonde-matrix with its transposed. What is left is a multiple of the Jacobian $n^{k} \prod \lambda_{i}^{n-1}$ of $\underline{\lambda}^{n}-\underline{a}$, the residue of which is known to be the degree $n^{k}$ of $\underline{\lambda}^{n}-\underline{a}$. Putting everything together we get the claimed normalization.

\section{REFERENCES}

[AsSa] A. Astashkevich AND V. SAdov: Quantum cohomology of partial flag manifolds $F_{n_{1} \ldots n_{k}}$, Comm. Math. Phys. 170 (1995) 503-528.

[BeMa] K. BeHREND, Y. MANin: Stacks of stable maps and Gromov-Witten invariants, Duke Math. J. 85 (1996) 1-60.

[Be1] A. Bertram: Towards a Schubert calculus for maps from a Riemann surface to a Grassmannian, Internat. J. Math. 5 (1994) 811-825.

[Be2] A. Bertram: Quantum Schubert calculus, preprint alg-geom 9410024, to appear in: Adv. Math.

[BDW] A. Bertram, G. Daskalopoulos and R. Wentworth: Gromov invariants for holomorphic maps from Riemann surfaces to Grassmannians, J. Amer. Math. Soc. 9 (1996) 529-571.

[CaDiSt] E. Cattani, A. Dickenstein, B. Sturmfels: Computing multidimensional residues, in: Proceedings of MEGA 1994.

[Fi] G. Fischer: Complex analytic geometry, LNM 538, Springer 1976.

[Fu] W. Fulton: Intersection theory, Springer 1984.

[GiKi] A. Givental AND B. Kim: Quantum cohomology of flag manifolds and Toda lattices, Comm. Math. Phys. 168 (1995) 609-641. 
[Gr] M. Gromov: Pseudo holomorphic curves in symplectic manifolds, Inv. Math. 82 (1985) $307-347$.

[GrHa] P. GRIFFITHS AND J. HARRIS: Principles of algebraic geometry, Wiley 1978.

[Ha] R. HARTSHORNE: Residues and duality, LNM 20, Springer 1966.

[In] K. Intriligator: Fusion Residues, Mod. Phys. Lett. A 38 (1991) 3543-3556.

[Ki] B. KIM: Quantum cohomology of flag manifolds $G / B$ and quantum Toda lattices, preprint alg-geom 9607001.

[LiTi] J. LI, G. TIAN: Quantum cohomology of homogeneous manifolds, J. Alg. Geom. 6 (1997) 269-307.

[Pi] S. PIUNIKhin: Quantum and Floer cohomology have the same ring structure, preprint hep-th 9401130.

[RRW] M.S. RAFI, J. Rosenthal AND X. WANG: Degree of the generalized Plücker embedding of a quot scheme and quantum cohomology, preprint hep-th 9402011.

[Ru] Y. RUAN: Topological sigma model and Donaldson type invariants in Gromov theory, Duke Math. Journ. 83 (1996) 461-500.

[RuTi] Y. RUAn, G. Tian: A mathematical theory of quantum cohomology, Journ. Diff. Geom. 42 (1995) 259-367.

[Ts] A.K. Tsikн: Multidimensional residues and their applications, Transl. of Math. Monographs 103, AMS 1992.

[Va1] C. VAfa: Topological Landau-Ginzburg models, Mod. Phys. Lett. A 6 (1991) 337-346.

[Va2] C. VAFA: Topological Mirrors and Quantum Rings, in: Essays on Mirror Manifolds (ed. S.-T. Yau), International Press 1992, 96-119.

[Wi1] E. Witten: Topological sigma models, Comm. Math. Phys. 118 (1988), 353-386.

[Wi2] E. WitTEN: On the structure of the topological phase of two-dimensional gravity, Nucl. Phys. B 340 (1990) 281-332.

[Wi3] E. WitTEN: The Verlinde algebra and the cohomology of the Grassmannian, preprint hep-th 9312104. 